ÉGYPTE monde arabe

\section{Égypte/Monde arabe}

30-31 | 1997

Les visions de l'Occident dans le monde arabe

\title{
Roumi et beldi
}

Réflexions sur la perception de l'occidental à travers une dichotomie locale

\section{Hassan Rachik}

\section{OpenEdition}

\section{Journals}

Édition électronique

URL : https://journals.openedition.org/ema/1656

DOI : 10.4000/ema.1656

ISSN : 2090-7273

\section{Éditeur}

CEDEJ - Centre d'études et de documentation économiques juridiques et sociales

\section{Édition imprimée}

Date de publication : 30 septembre 1997

Pagination : 293-302

ISSN : 1110-5097

\section{Référence électronique}

Hassan Rachik, «Roumi et beldi », Égypte/Monde arabe [En ligne], 30-31 | 1997, mis en ligne le 08 juillet 2008, consulté le 07 juillet 2022. URL : http://journals.openedition.org/ema/1656 ; DOI : https:// doi.org/10.4000/ema.1656

Ce document a été généré automatiquement le 7 juillet 2022.

Tous droits réservés 


\title{
Roumi et beldi
}

\author{
Réflexions sur la perception de l'occidental à travers une dichotomie \\ locale
}

\author{
Hassan Rachik
}

1 L'étude des représentations de l'Occident réfère généralement au discours savant et néglige maintes catégories de faits renseignant sur ce que les gens disent et font dans la vie de tous les jours. Notre approche s'inspire davantage de nos précédentes études sur le rituel et le sacrifice. D'abord, l'entrée dans l'univers des représentations part des termes vernaculaires et des notions qu'ils renferment. Notre point de départ consiste en une classification binaire locale où l'un des termes réfère à la notion de l'Occident. $\mathrm{Au}$ Maroc, en milieu rural et urbain, plusieurs produits de consommation courante (le savon, l'huile, le beurre, la levure, etc.) sont classés en roumi et beldi. Le mot roumi, qui dériverait de al-roum (Byzance), désigne le chrétien et tout ce qui est originaire de l'Occident. Le mot beldi signifie littéralement « du pays » (balad)et s'applique à tout ce qui est exclusivement « local » et « indigène ».

2 Le second ancrage de notre observation est constitué par des faits où la dichotomie étudiée est mise en acte. Ces faits se rapportent à l'organisation de l'espace domestique et aux comportements vestimentaires. De nouvelles pratiques d'ameublement distinguent le salon roumi du salon beldi' (dans le discours «savant", on emploie aussi les expressions salon européen/salon marocain). La même opposition est appliquée au niveau vestimentaire; on distingue alors Ibas roumi (le vêtement occidental moderne) et Ibas beldi (le vêtement traditionnel).

3 Au début des années cinquante, Villème, de passage à Fès, visite « un jeune ménage imbu de modernisme: notre hôte, en contact depuis plusieurs années avec les Européens, se déclare très affranchi des coutumes. Il n'a pas de préjugé contre les boissons fermentées et il est très fier de présenter sa femme aux hommes européens (mais pas aux marocains). Il occupe... une seule grande chambre meublée de la façon suivante : au milieu et face à la porte, un lit pour deux personnes, très moderne encadré de descentes de lit et petites tables de nuit avec lampe [...]. À droite, une salle à manger du style « Galeries Barbès ", d'un bois clair et luisant, chaises, buffet bas-ensemble très clinquant. À gauche, un salon traditionnel marocain (beldi),divans, coussins et table 
basse où nous est offert un repas très orthodoxe " (Villème, s.d., p. 69). L'hôte, moderniste et affranchi des coutumes marocaines, ne sert pas son invité français dans la salle à manger européenne (qui est juste à côté) mais au salon traditionnel. Tout l'ameublement de la maison est importé, sauf le salon marocain, qui, nous l'imaginons, est l'œuvre des artisans de Fès.

Lorsque les gens disposent d'un grand espace, le dilemme entre les deux modes d'ameublement est résolu en adoptant le salon marocain et le salon européen. "Même chez le bourgeois européanisé, la tradition résiste encore sur ce point. On rencontre souvent des intérieurs où toutes les pièces sont meublées à l'européenne, à l'exception du salon qui reste conforme au vieil usage : divans bas, chargés de coussins brodés, tentures murales de couleur, tapis de Rabat. Dans certaines maisons riches, il y a deux salons, l'un à la mode européenne, l'autre à la mode marocaine. De toute façon, s'il n'y a qu'une pièce qui demeure conforme à la tradition, c'est toujours celle-là » (Adam, 1968, p. 140) Parlant de juifs marocains qu'il considère comme désireux d'« adopter jusque dans ses moindres détails la vie européenne ", Villème remarquait que la « seule concession à la coutume ancienne est la coexistence très fréquente de deux salons : l'un meublé à l'européenne, l'autre à la marocaine... » (Villème, s.d., p. 82).

Répondant à la question de savoir s'ils souhaitaient aménager leur intérieur « entièrement à la mode traditionnelle, entièrement à la mode européenne, ou de façon mixte ", 93 \% de jeunes lycéens de Casablanca et de Fès (418 enquêtes) ont opté pour une solution mixte, $4 \%$ pour la mode européenne et $1 \%$ pour la mode traditionnelle (Adam, 1963, p. 107-108). Le compromis entre les deux modes d'ameublement n'est pas exclusif à la génération des pères. Même actuellement, et chaque fois que le budget domestique et l'espace habité le permettent, plusieurs jeunes couples perpétuent le même compromis.

6 Comprendre la raison d'être de ce compromis, c'est saisir les significations et les usages des deux modes d'ameublement. Le salon roumi est arrangé à l'occidentale, il est généralement garni d'un canapé, de deux fauteuils et d'une table centrale (occasionnellement de petites tables latérales, de coussins, etc.) Le salon beldi reproduit de façon ostentatoire l'ameublement traditionnel de la pièce principale de la maison ; il se compose de banquettes couvertes de matelas et de coussins, le tout adossé au mur et faisant le tour de la pièce. Le salon beldi est consommateur d'espace, il n'admet pas la concurrence d'autres meubles tels que l'armoire, le buffet-vitrine ou la bibliothèque. Pour accentuer l'aspect beldi du salon, on ajoute du zellige (mosaïques incrustées dans le mur) ${ }^{1}$. Parfois, l'usage du stuc constitue la touche finale : il s'agit d'une composition de plâtre sculpté ornant le plafond ou courant en frise sur la partie supérieure du mur. Cette décoration peut être aussi en bois sculpté de motifs traditionnels.

7 Le salon roumi est généralement le coin intime de la famille où l'on regarde la télévision, où l'on écoute de la musique. C'est aussi le lieu où l'on reçoit amis et parents lorsqu'ils ne sont pas nombreux. En principe, on n'y mange pas, tout au plus y sert-on des boissons. À l'opposé, le salon beldi est souvent inoccupé, voire désolé. Il est ressuscité pendant les fêtes et les réceptions cérémonielles où il est exhibé, admiré.

8 L'introduction du salon roumi a créé un redoublement d'objets (deux types de salons) et par là même une adaptation du dispositif mobilier «traditionnel ». Celui-ci ne recevait pas le nom de salon beldi, qui est récent et qui n'existe que par opposition au salon roumi. Le dispositif précédent, composé des mêmes éléments, était désigné par un terme qui ne référait à aucune dichotomie : bitdiyaf, la chambre des invités. Nommer 
une pièce de la maison était une manière d'indiquer sans ambages sa fonction. Ce qui n'est pas le cas de l'expression "salon beldi", qui est équivoque. Elle est composée de deux mots qui normalement doivent s'exclure. Au Maroc, un salon est forcément moderne. Derrière le mot beldi, il faudrait identifier des changements dans les perceptions de l'Occident et du patrimoine traditionnel.

Pourquoi alors adopter une nouvelle expression pour désigner un mode d'ameublement ancien? C'est que le salon beldi, contrairement à la «chambre des invités ", n'est pas isolé, il partage le même espace, la réception, avec le salon roumi. La réception est souvent « un espace semi-ouvert, sans cloisons mais avec des repères plus ou moins fixes comme demi-murets, moucharabieh, différences de niveau, qui permet de voir ou d'entrevoir deux ou trois sous-espaces différents : "un salon marocain » (beldi ou taqlidi en arabe classique) garni donc de banquettes faisant le tour de la pièce ; un "salon moderne » (encore appelé européen), garni de canapés et fauteuils, un coin de feu, espace petit, intime... » (Navez-Bouchanine, 1988, p. 287). La position qu'occupe le salon be/d/est nouvelle. Son nom et sa signification sont fonction d'une contiguïté avec un mode d'ameublement occidental. C'est au moment où de riches citadins ont voulu meubler un grand espace (grands appartements et villas) qu'est apparue l'idée d'un salon beldi, construit par opposition au salon roumi, introduit au Maroc par les Européens.

10 En fait, la nouvelle expression «salon beldi» ne désigne pas un mode d'ameublement ancien et traditionnel mais une cohabitation récente entre deux modes d'ameublement répondant à deux fonctions différentes. C'est une négociation délicate opérée par des citadins qui ne peuvent ou ne veulent renoncer ni à la «tradition" ni à la «modernité ». Mais la négociation et le compromis se font selon une nouvelle vision du traditionnel et de l'occidental. On adopte l'ameublement occidental parce qu'il est quotidiennement fonctionnel sans sacrifier la dimension esthétique et rituelle de l'organisation domestique de l'espace.

11 Un cas limite illustre mieux cette association entre l'européen et le fonctionnel d'une part, et le traditionnel et le cérémoniel, d'autre part. Un riche commerçant de Fès a construit, au début des années cinquante, deux maisons dans son vaste jardin boisé. «L'une des maisons est de style traditionnel flamboyant. [Elle est] destinée à offrir, dans un cadre d'opulence extrême, de brillantes réceptions tant aux industriels et commerçants européens... qu'aux grandes familles marocaines à qui il convient d'affirmer sa puissance. L'autre maison, d'une conception bien plus moderne, est européenne tant par sa forme que par son ameublement. Habitée en permanence par le maitre de maison, elle est, lors des grandes réceptions, réservée aux femmes que ne gêne pas, grâce au jardin, la proximité des étrangers » (Villème, s.d., p. 80) Ce n'est pas seulement la réception qui est divisée en une partie traditionnelle et une autre moderne, mais tout l'espace habité.

On assigne au roumi, à l'occidental, une dimension technique, utilitaire, et au beldi une dimension, cérémonielle, symbolique et affective. C'est comme si, par beldi, on tendait à assigner une limite à une occidentalisation intégrale. Il existe des domaines où le beldi, revu et repensé, est plus approprié. Le domaine rituel est tout indiqué. Le salon marocain est un espace cérémoniel, un espace auxiliaire qui vient au secours du salon européen lorsque le nombre d'invités, pendant les fêtes et les cérémonies, excède la capacité d'accueil de ce dernier. 
13 Le couple beld-roumi, comme d'autres couples classificatoires, induira en erreur si on maintient séparés les éléments qui relèvent de chaque classe. Il ne s'agit pas d'une juxtaposition de deux types d'ameublement mais d'une nouvelle structure d'ameublement, même si les éléments qui la composent ont des fonctions et des significations différentes dans des contextes antérieurs. Il est fort possible que, lorsqu'il existe en plusieurs exemplaires, l'un des objets se libère de sa fonction objective au profit d'un usage symbolique (Thysen, 1983). Le salon beldi est souvent réduit à sa dimension rituelle et esthétique. La fonction utilitaire quotidienne est assurée par le salon roumi.

L'ameublement en beldi et roumi nécessite un grand espace. Peu de gens en fait ont la possibilité de posséder les deux types de salon². Mais pour ceux qui en ont les moyens, le choix de posséder plusieurs salons européens est une possibilité qui est rarement observée. Le redoublement d'objet ne serait donc pas seulement motivé par des raisons ostentatoires. Par ailleurs, il n'est possible que dans la mesure où les objets anciens sont valorisés. Et dans notre cas, valoriser un objet ancien, c'est lui assigner une fonction rituelle et cérémonielle. Ceci est encore avéré sur le plan vestimentaire, où le choix des pratiques est largement plus grand.

Les femmes citadines portaient traditionnellement un haik, vaste pièce de cotonnade blanche dans laquelle la femme se drapait pour sortir. À partir des années cinquante, le haik commence à être sérieusement concurrencé avant d'être supplanté, dans plusieurs villes du Maroc, par la djellaba. Celle-ci s'est imposée malgré la résistance des nationalistes et des autorités marocaines qui la trouvaient non conforme à la tradition. Le pacha de Fès prenait même des sanctions pénales contre les femmes qui persistaient à la porter (Villème, s.d., p. 61).

La djellaba subit ensuite plusieurs changements. «D'abord absolument classique et simplement empruntée à l'homme, la djellaba commence elle-même à évoluer; en effet, depuis deux ou trois ans, on assiste (fin des années quarante) à la naissance d'une véritable mode créée à Fès et les élégantes se mettent à porter des djellabas à empiècement et emmanchures montées avec épaulettes à la façon des tailleurs féminins modernes, rangées de boutons sur le devant [...]. C'est là quelque chose de très nouveau, un véritable essai de modernisation d'un vêtement typiquement marocain. » (Villème, s.d., p. 61) Le changement le plus important, parce qu'il touche non seulement la forme mais aussi la structure de la djellaba, se situe au début des années soixante : de jeunes femmes ont opté pour une djellaba sans capuchon et sans voile ${ }^{3}$.

17 Parallèlement aux changements que connaît le costume traditionnel citadin, l'adoption du costume européen se diffuse principalement chez les filles scolarisées et, plus tard, chez les femmes travaillant en dehors du foyer (employées et fonctionnaires notamment). Le Ibas roumi s'applique à tous ces vêtements récemment introduits que seules des femmes occidentales portaient (jupe, robe, etc.). Le Ibas beldi s'applique aux vêtements traditionnels qui sont produits de façon artisanale (djellaba, cafetan, qmiss, etc.). Le costume des hommes le plus répandu était la djellaba (vêtement de dessus à manches courtes muni d'un capuchon), le burnous (vêtement d'extérieur, cape très large et sans manches munie d'un capuchon) et le haïk (Besancenot, 1988, p. 139 ; Laroui, 1977, p. 31). La chaussure la plus répandue, notamment en ville, est la balgha (babouches). Comme coiffure, le turban (rezza)était généralement utilisé. C'est une "longue pièce de tissu que l'on porte enroulée autour de la tête en savantes torsades " (Villème, s.d., p. 58) ${ }^{4}$. 
18 À noter que, pendant la colonisation, même ceux qui adoptaient le costume européen faisaient une concession au niveau de la coiffure. C'est le fez, nommé localement tarbûsh, de lainage rouge agrémenté d'un gland de soie noire (Besancenot, 1988, p. 143) qui devient la coiffure du citadin moderne ${ }^{5}$. En portant le costume européen sans renoncer au fez, l'élite citadine cherchait à se distinguer des non-musulmans. Les membres des gouvernements du Maroc ont continué à le porter pendant les premières années qui ont suivi l'indépendance. Aujourd'hui, le fez n'est généralement porté qu'avec la djellaba.

19 Excepté cette phase de colonisation, le costume européen actuel (ce qui n'est pas le cas $\mathrm{du}$ salon européen) est exclusif et n'admet pas la concurrence des pièces traditionnelles. Il en était de même du costume traditionnel. La djellaba par exemple se portait généralement avec un saroual et des babouches et rarement avec un pantalon long et des chaussures modernes. Mais il arrive souvent que des hommes et des femmes qui dans la vie de tous les jours portent un costume européen disposent, chacun selon ses moyens, d'un « vestiaire traditionnel ». Comme pour le salon, l'usage du Ibas beldi est de plus en plus réservé aux contextes rituel et cérémoniel généralement liés aux fêtes familiales et religieuses. C'est dans ces occasions que les femmes portent des vêtements traditionnels. Le cafetan (une sorte de robe ample et longue, sans col, à manches relativement larges), et d'autres habits du même genre (takchita, qmiss)sont appropriés au temps festif. Contrairement à la djellaba, le cafetan est porté avec des chaussures modernes (les babouches comme le cherbil, plus sophistiqué, ne sont portés que par les femmes d'un certain âge). Le deuil est aussi un contexte où le vêtement roumi est fortement déconseillé. Pour exprimer sa tristesse et présenter ses condoléances, une femme doit porter une djellaba simple, souvent sans capuchon.

Du côté des hommes, la djellaba est de plus en plus valorisée. Elle est souvent portée le vendredi (jour de prière collective) et les jours de fête. La norme est plus souple. Les hommes se présentent souvent dans les fêtes et lors d'un deuil en costume européen.

21 Le Ibas beldi, qui faisait intégralement partie de la quotidienneté des Marocains, tend à être associé aux fêtes et cérémonies traditionnelles. La dichotomie beldi/roumifonde de plus en plus la séparation entre le quotidien et l'utilitaire, d'une part, et le festif et le rituel, d'autre part. Pour les gens qui, quotidiennement, s'habillent à l'occidentale, porter le Ibas beldi relève du cérémoniel. Cependant, pour perpétuer une tradition, il faudrait la rénover, l'adapter et lui assigner une fonction rituelle et cérémonielle avant qu'elle ne soit oubliée, ou, dans le meilleur des cas, figée dans un musée ${ }^{6}$.

22 C'est au prix d'une sélection que le costume traditionnel, travaillé par la mode européenne, trouve son dernier refuge dans le cérémoniel. Il assume sa nouvelle fonction en s'adaptant à la mode vestimentaire actuelle (vêtement de moins en moins ample, de plus en plus léger, etc.). Nous retrouvons les mêmes suggestions de John Drummond Hay, lorsqu'il conseillait aux militaires marocains du siècle dernier (1855), de se débarrasser « des larges robes qui entravaient leurs mouvements et d'adopter des vêtements qui les habillaient plus près du corps, gagnant ainsi une plus grande liberté dans l'usage de leurs membres » (cité par Laroui, 1977, p. 30).

23 Toutes les pièces du costume traditionnel n'ont pu être adaptées à la nouvelle fonction cérémonielle. Ni le cafetan, ni la faragia (particulièrement portée par les lettrés et les commerçants citadins) ni le haik, etc., n'ont pu coexister avec le vêtement européen (Besancenot, 1988, p. 140 et s.). 

rigoureuse aux goûts modernes. Le salon beldi n'est qu'un avatar du salon traditionnel. Celui-ci est une pièce qui a «la forme d'un rectangle allongé. La largeur (env. 2,80 m) correspondant au maximum de ce qui pouvait être obtenu en utilisant la technique des plafonds portés par travée de bois. Avec l'introduction de la dalle de ciment, la dimension tend à changer.» Le salon beldi «est plus carré que rectangulaire. D'inspiration très largement traditionnelle, il s'éloigne pourtant souvent, en matière de décoration, dudit modèle : choix de tissus, hauteur et largeur des banquettes, éléments de décoration murale, etc. » (Navez-Bouchanine, 1988, p. 287). adapter le salon traditionnel. L'une de ses pages publicitaires présente une vision plus occidentale du salon traditionnel. Elle commence par en souligner les défauts :

« Votre salon marocain ne vous satisfait plus. Vous êtes las de ces divans si hauts où l'on est plus perché qu'assis, si lourds qu'il faut se mettre à plusieurs pour en déplacer un et si aisément déformables que l'on est obligé sans cesse d'en retendre le tissu. Vous redoutez le moment inévitable où il faudra tout découdre pour laver et sécher la laine. Enfin et surtout, vous en avez assez d'avoir dans votre maison un espace perdu, une pièce si peu utilisée et déserte à longueur d'année. Il est peut être temps pour vous de découvrir la banquette à ressort « Impériale » de Richbond, car dans bien des maisons déjà, elle a transformé le salon marocain en une pièce où il fait bon vivre tous les jours en famille et avec ses amis. Pas trop haute pour un confort maximum et très légère, elle est faite de ressorts en acier trempé indéformable qui lui confèrent une tenue et une longévité exceptionnelles. »

Avec les grands fabricants d'ameublement, la frontière entre les deux modes d'ameublement devient ambiguë. Avec des arguments fonctionnels, le salon marocain se rapproche du salon européen. Le fabricant moderne entend enterrer la raison d'être du salon traditionnel. On reproche au matelas traditionnel d'être si haut, comme si notre riche citadin ne pouvait mettre moins de laine. Le salon beldi est d'abord prestigieux, et la chère laine en est l'élément principal, et plus il y a de laine, plus on est perché.

On reproche au matelas traditionnel d'être lourd, mais dans la maison du riche, les domestiques ne manquent pas. On reproche aussi au salon traditionnel d'être désolé, mais que faire des grands espaces qu'on ne peut pas totalement et quotidiennement occuper? On oublie que le salon beldi estassocié à une logique d'abondance et de fête. Avec la logique actuelle de la rareté et du calcul, on va s'accommoder des matelas de série, mais on va continuer à leur appliquer le même nom ancien.

Que reste-t-il de l'ameublement et des vêtements qualifiés de beldi lorsque de nouveaux cadres spatio-temporels, de nouvelles fonctions et significations leur sont assignés? Le nom, certainement ; la joie, comme dans toute fête, d'accentuer l'aspect esthétique et la séparation d'avec un quotidien dominé par l'utilitaire ; la jouissance du collectionneur qui exhibe des pièces de sa chère collection; l'illusion d'entretenir une spécificité et de brandir une identité... ${ }^{7}$ 


\section{BIBLIOGRAPHIE}

ADAM A :

- 1968, Casablanca. Essai sur la transformation de la société marocaine au contact de l'Occident, 2 tomes, Paris, CNRS.

- 1963, Une Enquête auprès de la jeunesse musulmane du Maroc, Aix-en-Provence, Publications des Annales de la faculté des lettres.

BESANCENOT J., 1988, Costumes du Maroc, Rabat, Al-Kalam.

BRUNOT L, 1923, « Les noms des vêtements masculins à Rabat », Mélanges René Basset.

ETIENNE Jean d', s.d., « Une famille Marocaine », Paris, Cahiers de l'Afrique et de l'Asie.

DEPAULE J.-C, 1989, « Un objet technique dans l'espace domestique », dans Espaces maghrébins. Pratiques et Enjeux, Oran, Enag/Editions-URASC.

GABRIEL-ROUSSEAU, 1938, Le Costume au Maroc, s.l.

LAROUI A., 1977, Les origines sociales et culturelles du nationalisme marocain (1830-1912), Paris, Maspero.

NAVEZ-BOUCHANINE F., 1988, « Modèles d'habiter : usage et appropriation de l'espace dans les quartiers résidentiels "de luxe" au Maroc », dans BADUEL R., Habitat, État, Société au Maghreb, Paris, CNRS.

THYSEN X., 1983, Des manières d'habiter dans le Sahel tunisien, Paris, CNRS.

VILLÈME Louis, s.d., « L'Évolution de la vie citadine au Maroc », Paris, Cahiers de l'Afrique et de l'Asie.

\section{NOTES}

1. «Cette famille très évoluée, une des rares dont les jeunes filles aillent au lycée, sortent dévoilées et assistent aux réceptions, habite une vaste maison où l'on voit des salons traditionnels aux murs couverts jusqu'à deux mètres du sol d'une surabondance de zelliges [...]. » «Les riches notables qui construisent des maisons à allure traditionnelle apprécient la surabondance de décor: les zelliges, jadis limités aux côtés des portes ou au pourtour des fenêtres envahissent les murs, les sculptures de plâtre recouvrent parfois des plafonds entiers. » (Villème, s.d., p. 69 et 71)

2. Dans les couches sociales modestes, « e mobilier est strictement traditionnel, l'armoire n'a pas supplanté le coffre, le fauteuil ni la chaise n'ont fait leur apparition, la table à manger reste petite, ronde, basse et de bois grossier, les ustensiles de cuisine et le service de table n'ont encore rien emprunté aux mœurs européennes, la natte de jonc recouvre seule le parquet " (d'Etienne, 1950, p. 29). Actuellement, le plus courant en ville est la pièce principale meublée modestement comme un salon beldi. Personne n'ose l'appeler ainsi, c'est une pièce qui répond à plusieurs usages (on y mange, on y dort et, faute de mieux, les gens sont souvent obligés d'y recevoir).

3. La djellaba sans capuchon était présentée comme l'une des causes de la sécheresse du début des années soixante. Je me rappelle des manifestants qui scandaient dans la rue : «jellaba bla qoubb (djellaba sans capuchon), ma khellat chta tsebb (empêche pluie de tomber) ». 
4. La taguiya, elle, est une coiffure qui semble avoir tardivement intégré le costume traditionnel. C'est une calotte de laine tricotée à l'aiguille.

5. «Le tarbouch, introduit d'abord dans l'armée, ne gagnera les couches urbaines que tout à fait à la fin du siècle. » (Laroui, 1977, p. 32)

6. Nous venons de commencer une étude des images des manuels scolaires officiels en fonction de nos questions relatives aux types de vêtements. Voici quelques résultats partiels. Nous avons recensé, dans un manuel de lecture en arabe ( $4^{\mathrm{e}}$ niveau primaire) 81 images en rapport avec le costume. Nous avons distingué trois catégories d'image : a) image représentant une ou plusieurs personnes ne portant que des vêtements traditionnels; $b$ ) image représentant une ou plusieurs personnes ne portant que des vêtements modernes ; c) image où les deux types de vêtement sont représentés. On dirait que les rédacteurs du manuel n'ont pas laissé au hasard la fréquence des images représentant le vêtement beldi et roumi. L'équilibre entre les deux types de vêtement est presque parfait. La moitié des images (46\%) représentent des personnes vêtues en beldi et d'autres en roumi alors que la première et la deuxième catégorie représentent respectivement $24 \%$ et $30 \%$. Toutefois les enfants portent souvent le vêtement occidental. Sur 52 images, seulement 4 représentent des enfants (dont 3 en milieu rural) avec un vêtement traditionnel. Le seul enfant citadin habillé de manière traditionnelle est une fille impliquée dans la cérémonie du henné. Seul le cérémoniel justifie le recours au vêtement beldi. Excepté le contexte cérémoniel, l'enfant est présenté comme occidentalisé sur le plan vestimentaire. Par contre, les images représentant des adultes consacrent une large place au vêtement traditionnel (47 images sur 91). 7. Ceci est proche d'un discours savant qui recommande d'adopter la technique occidentale (conçue comme universelle) tout en sauvegardant une spécificité marocaine réduite à des éléments de la culture (langue, religion, coutumes).

\section{INDEX}

Mots-clés : beldi, Maroc, Occident, roumi

\section{AUTEUR \\ HASSAN RACHIK}

Université Hassan II, Casablanca 